

\title{
Geotomography applied at the Stripa Mine in Sweden
}

\author{
J. T. Okada \\ E. F. Laine \\ R. J. Lytle \\ W. D. Daily
}

Manuscript date: April 7, 1980

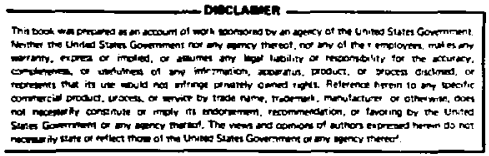

\section{LAWRENCE LIVERMORE LABORATORY \\ University of California - Livermore, California $\bullet 94550$}




\section{CONTENTS}

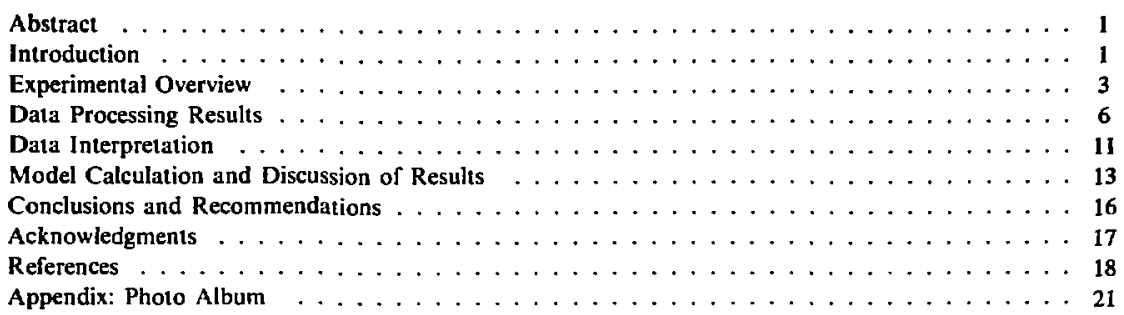




\section{Geotomography applied at the Stripa Mine in Sweden}

\section{ABSTRACT}

LLNL made uitrahigh-frequency (450-MHz) eleciromagnetic borehole-toborehole transmissions in the Stripa Mine near Guldsmedshyttan, Sweden, in April 1979. Transmission loss measurements were made between four sets of $76-\mathrm{mm}$ boreholes $30 \mathrm{ml}$ long, located at the end of a drift. Distances between the boreholes varied from 2 to $22 \mathrm{~m}$. More than 25,000 data points were taken. A geotomograph was constructed to show the variation of attenuation between boreholes. The observed spatial variation of electromagnetic attenuation may be related to the expected stress relief created by the mine. Transmission loss measurements were also made between two converging boreholes drilled from the surface to points near the mine. Data taken at $21 \mathrm{MHz}$ show a nearly uniform attenuation within this sampled region. These experiments demonstrate that geotomographic data collection/interpretation provides high-resolution images of the underground environment and can provide useful input to those charged with providing the detailed site characterizations needed for both short- and long-term monitoring of underground nuclear waste repositories.

\section{INTRODUCTION}

A cooperative effort between the Swedish Nuclear Fuel Supply Company and the Lawrence Berkeley Laboratory of the U. S. Department of Energy was formed in 1977 to study the feasibility of s:on: $:$ radioactive wastes in mined granite caverns. ${ }^{1}$ Scientists from both countries selected as the project site the Stripa Mine, an abandoned iron ore mine near the lown of Guldsmedshyttan, Sweden. The Stripa Mine is locilled some 200 kilometers west of Stockholm (see Fig. 1). The mine, originally opened in 1485 , was closed and reopened several times over the next five centuries, and continued to produce ore until late 1976. Its exceller t operating conditions, accessibility, and physical similarity to granite caverns in the United States contributed to its selection as an ideal experimental facility. This site has enabled researchers to study if granite calverns have the potential to safely jsolate radioactive wastes from man and his environment.

Dr. Paul A. Witherspoon, Associate Director of the Lawrence Berkeley Laboratory, who has been instrumental in recogrizing the need for this work, has stated that "resolving the problem of radioactive waste storage is crucial to the future use of nuclear energy throughout the world." ${ }^{\text {T }}$ To address part of the crucjal waste storage problem, extensive rock mechanical, hydrological, and geophysical measurements have been performed at the Stripa Mine site. ${ }^{2}$ The major experiments at the mine are to simulate the thermal load induced by radioactive waste containers and examine the effects of thermal stress on the rock mass. A number of conventional geophysical tools, such as seismic wave-form, neutron, gamma-gamma, natural gamma, caliper, and electrical resistivity, have been deployed to detect fractures, measure the witer content, and supplement the geological information. This report describes the results of a Lawrence Livermore National Laboratory deployment at this site of a new gcophysical technique: high-frequency clectromagnetic geophysical tomography (geolomography).

Gcolomography is a concept conceived and developed at the Lawrence Livermore National Laboratory. ${ }^{3,4}$ It is a geophysical data collection and interpretation procedure similar to that used in $x$-raly tomography, 5.6 which is commonly used for biophysical imaging. The basic data collection concept is shown in Fig. 2. A cross section of the 


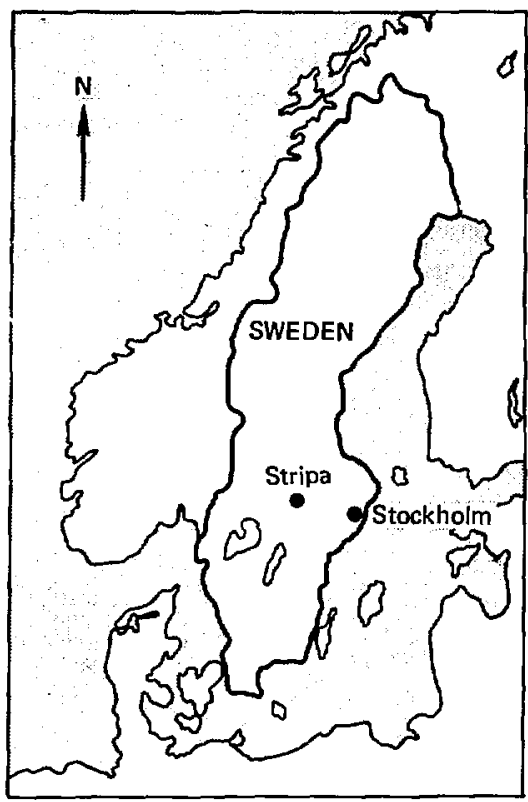

FIG. 1. The experimental site is located at the Stripa Mine, sbout $200 \mathrm{~km}$ from Stockholm, Sweden.

ground is probed in detail, either seismically or clectromagnetically, by measuring the transmission characteristics (signal attenuation and time of arrival) between numerous source and receiver pairs. The Stripa effort utilized high-frequency electrotnagnetic energy, measuring signal attenuation to characterize the medium. The properties of a medium affect the waves passing through it in a mathematically describable way. By appropriately describing this mathematics and performing the suitable inversion it is possible to generate a pseudocolor or gray-level image of the geophysical environnent through which the waves passed. With a suitably large number of transmission paths and view angles, it is possible to generate highresolution images of the geophysical environment existing betwcen coplanar boreholes. These images can depict the electromagnetic or seismic constitutive parameters of the medium. An cxample of such an image is given in Fig. 3.

LLNL has successfully employed this technique for a range of applications and environments.
Situations have included characterizing a prospective urban transit station area prior to excavation,? assessing the quality of a bridge foundation seal, ${ }^{8}$ and defining the location and extent of grout pumped into boreholes at a construction site. ${ }^{9}$ In most of these situations, conventional techniques either would not have been suitable or would not have provided the detailed information desired. $\mathbf{A}$ major limitution of conventional techniques such as core sampling or logging is that they only provide detailed information on what is in the very near vicinity of boreholes. With widely separated boreholes, these techniques can furnish little detailed knowledge of the region between boreholes. A major advantage of the geotomography approach is that detailed knowledge of the region between boreholes is systematically collected and interpreted. In addition, the results are easily put into an intelligible form (i.e., an image), rather than in the common experimental geophysical format of squiggly lines.

To assess the utility of the geotomographic approach for providing knowledge needed in studying the leasibility of storage of radioactive wastes in mined granite caverns, LLNL sent a three-man team to Stripa for five weeks. The experiment and interpretation of the results constitute the body of this report. The appendix depicts the physical environment in which the experiment was conducted.

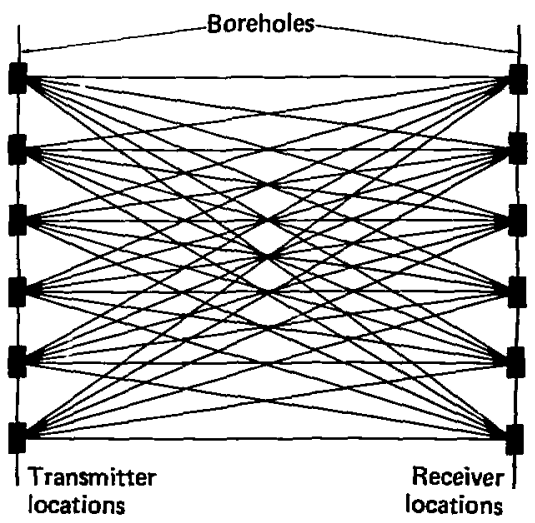

FIG. 2. Cross-borehole transmissions utilize multiple locations for the transmitters and receivers. 


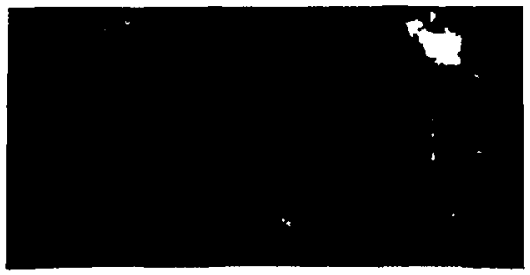

(a) Color mapping

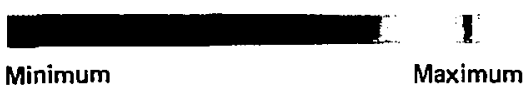

(b) Calibration
FIG. 3. The variation of electromagnetic attenuation between boreholes can be represented by a pseudo-color mapping. An example of such an inage is shown in (a). using the calibration representation shown in (b). Gray-level mapping can also be used.

\section{EXPERIMENTAL OVERVIEW}

The geotomography experiments at Stripa consisted of measurements in boreholes entering the ground from two locations-one at the surface and the other inside a mine drift. The equipment used to perform the measurements consists of standard electronics equipment (see Fig. 4) and antennas of LLNL design and construction. For the in-mine measurements the transmitting and receiving antennas were identical $25-\mathrm{cm}$ bicone structures with coaxial center feeds. Eight radially symmetric wires approximated the bicone. The total flare angle was approximately $20^{\circ}$. The surface measurements used a 1-m bicone transmitting antenna, coaxial center feed, eight-wire approximation, and total flare angle of approximately $5^{\circ}$. The receiving antenna for the surface measurements was a physically short, $25 \mathrm{~cm}$, active monopole. Power levels, measured at the out put of the power amplifier, were 11 and $76 \mathrm{~W}$ for the mine and surface boreholes, respectively.

The major part of the effort was conducted at the face of the ventilation drift of the mine, located approximately $330 \mathrm{~m}$ beneath the ground surface. Five boreholes, shown in the upper right-hand corner of Fig. 5, were drilled into the granite to provide a number of coplanar boreholes from which we could remotely probe the environment near the drift. These boreholes were $76 \mathrm{~mm}$ in diameter, and the distance between them varied from 2 to $22 \mathrm{~m}$. Measurements were performed on $450-\mathrm{MHz}$ transmissions from borehole $\mathrm{HI}$ to $\mathrm{H} 5, \mathrm{H} 2$ to $\mathrm{H} 5$,

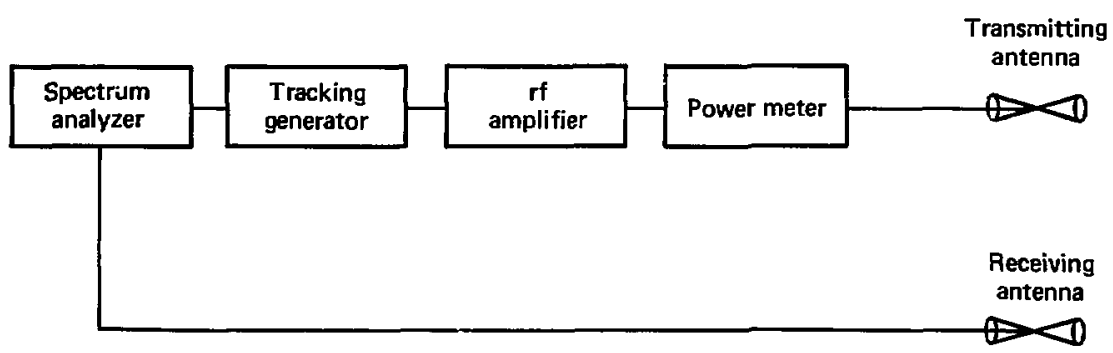

FIG. 4. The mensurement system comsists of monitoring the difference between the power transmitted by the trassaitting antenas and that received by the receiving antenna. 


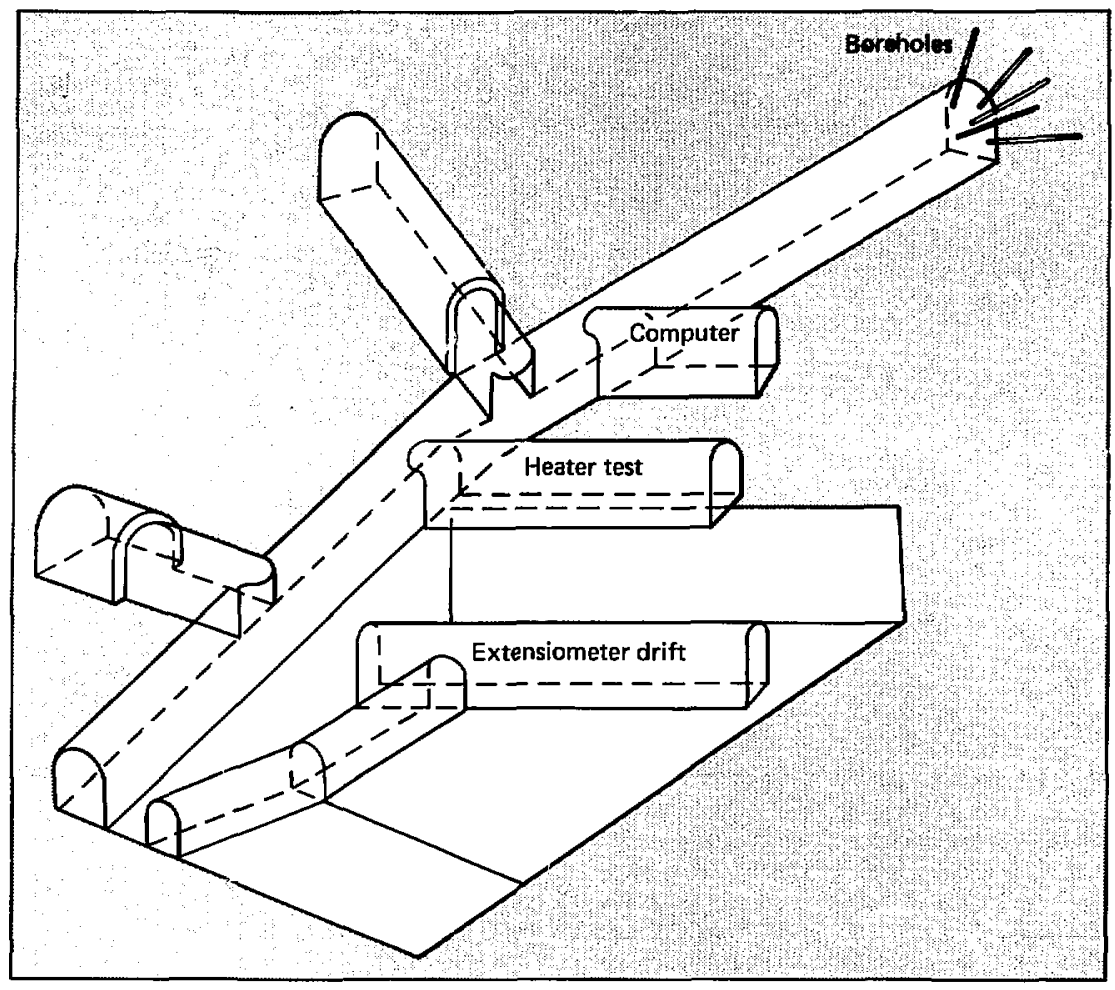

FIG. 5. The mine drifts at the experimental level ( $330 \mathrm{~m}$ depth). Boreholes $\mathrm{H1}, \mathrm{H2}, \mathrm{H3}, \mathrm{H4}$, and $\mathrm{H5}$ are at the end of the drift in the upper right-hand comer of the picture.

$\mathrm{H} 1$ to $\mathrm{H} 3, \mathrm{H} 1$ to $\mathrm{H} 2$, and $\mathrm{H} 2$ to $\mathrm{H} 3$ (see Fig. 6). We sampled various sectors between these boreholes, along sections varying in length from about $11 \mathrm{~m}$ to more than $20 \mathrm{~m}$. (Actual dimensions and shapes of the sampled sections are shown along with the sampling data in the following section, "Data Processing Results.") The selection of the dimensions of each section was constrained by the limited time available for our total experimental deployment. The interim distance between two consecutive source or receiver locations within a borehole was fixed at $20 \mathrm{~cm}$. This meant that each sector sampled had a minimum of more than 2900 combinations of source and receiver locations. The maximally sampled sector (H1 to H5) had 12,000 source-receiver combinations.

Prior to collecting these extensive data within the mine, which took four weeks using our manual placement and recording system (see appendix), differential transmission measurements ${ }^{10,11}$ were performed to assess the gross values of electrical permittivity $\epsilon$ and electrical conductivity o. These measurements were performed at $450 \mathrm{MHz}$ at a variety of locations within boreholes $\mathrm{HI}$ and $\mathrm{HS}$. 


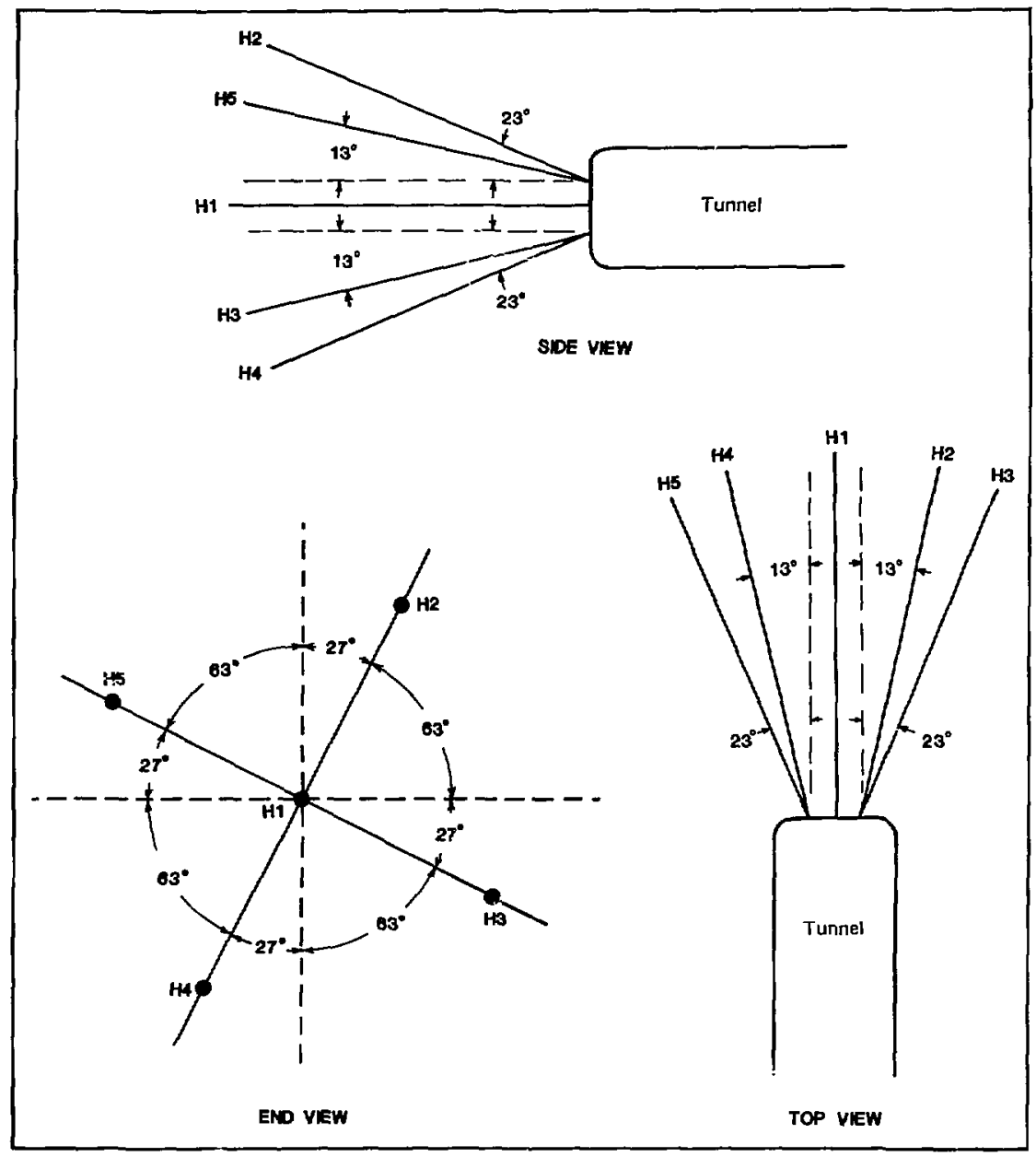

FIG. 6. The side, end, and top views of boreholes $\mathrm{H1}, \mathrm{H2}, \mathrm{H3}, \mathrm{H4}$, and $\mathrm{H5}$ shown relative to the end of the mine drift.

The results indicated nominal values of relative dielectric constant $\epsilon_{r}=4$ and electrical conductivity $\sigma=0.0015 \mathrm{~S} / \mathrm{m}$.

At the end of our deployment, a few days' effort was expended to perform measurements in two noncoplanar boreholes drilled from the ground sur- face. These boreboles converge and almost intersect near the mine. The separation between them is approximately $500 \mathrm{~m}$ at the ground surface and approximately $30 \mathrm{~m}$ near the mine. The boreholes come to within $50 \mathrm{~m}$ of the mine. Even though the weather conditions were inclement at the time of 
deployment and our probing system is at a rudimentary stage of development, we performed cross. borehole propagation to attempt to define the subsurface environment and to show that cross- borehole probing can be performed over significant distances in environments of waste disposal interest. Results for this set of boreholes from the ground surface are also given in the following section.

\section{DATA PROCESSING RESULTS}

Conceptually the procedure employed to obtain a gcotomograph from the data taken is straightforward. One starts with the data, a set of Idimensional estimates of the medium loss. By com. bining this information in a self-consistent manner, a 2-dimensional picture of the medium loss can be generated. The data are obtained by transmitting an electronagnetic signal, allowing the signal to propagate through the medium, and then detecting the signal. The measured quantity of the signal is the power level. The difference between power transmitted and that received, after the spatial loss is accounted for, is the loss attributed to the medium along that straight line path from transmitter to receiver.

The matter would be trivial if antennas were ideal isotropic point transmitting and receiving structures. Since they are not and an independent measurement of the antenna radiation pattern in situ is difficult, we used the transmission data themselves in an attempt to minimize antenna pattern effects. To describe this procedure, we first discuss the situation of a homogeneous medium.

For a perfectly homogeneous medium, any interpreted differences in the medium loss for different paths must be attributed to some change in the experimental conditions and not to the medium. If care is taken so that the only change in the experimental conditions which could affect the medium loss esitimate is the orientation of the transmitting and receiving antennas, then the loss as a function of that orientation can be estimated. With this information the composite transmitting/receiving antenna pattern can be obtained. Thus the strategy is: 1) transform the region sampled into one that is as nearly homogeneous as possible, 2) estimate the antenna pattern factor from this transformed region, 3) remove the antenna pattern factor effects from the data, 4) retransform the region (by an inverse of the procedure which made it homogeneous), and
5) calculate the geotomograph of the region. The only open question left is what transform is needed to produce a homogeneous region.

Under certain conditions it may be reasonable to assume that the region is homogeneous or that all a priori knowledge of the region is such that no prejudices are more reasonable than others. Then the best estimate for the pattern factor can be obtained with no transformations required. However, in the case of the Stripa measurements, since the region sampled is located in the vicinity of mining activity, an effect on the medium due to the action of forming the mine could be present. It is reasonable to expect that closer to the minc the effect might be different from what it is farther away. To test this hypothesis, it is necessary to examine the data for such a trend. Choosing the proper subset of the data, $i . e .$, those ray paths parallel to the mine face, a test can be made to see if there exist trends in thc medium attenuation with distance from the mining activity. Analyzing the data in this manner for all the planes investigated revealed a monotonically decreasing medium attenuation as a function of distance from the mine face. By using the above information, the trend found can be removed from all the data, with the result that the region now is as homogeneous as possible, consistent with the information known about the region. Since for this particular situation there are no other known mechanisms or conditions which might be exploited to ascertain additional inhomogeneities, this is the best that can be done and the antenna pattern effects can be estimated from the transformed data.

For all the planes depicted by a geotomograph, the general character of the medium loss is a monotonically decreasing function of distanee from the mine, with a greater rate of change closer to the mine face. There are small variations in the loss along lines parallel to the mine face; however, the predominant effect is the above-mentioned monotonic behavior. At distances greater than $20 \mathrm{~m}$ 
from the mine face (at least four tunnel diameters), the medium loss approaches a constant (see Figs. 7 and 8). As expected, the character of the geotomographs is consistent with the information used to estimate the antenna pattern effects. In summary, our data for transmission between boreholes H1 and H5 show that the electromagnetic loss characteristic of the rock near the tunnel decreases gradually with distance from the mine face.

It was not possible to easily generate a geotomograph for the plane between the boreholes from the surface, as they are not coplanar. However, we were able to perform transmissions between these two boreholes. The regions explored are shown in Fig. 9. Note that transmissions were sent over a distance of $335 \mathrm{~m}$. Extensive measurements were taken for slant-line depths greater than
$310 \mathrm{~m}$. By ignoring the fact that these boreholes from the surface are not coplanar, we were able to process the data using the same approach as used for the boreholes from within the mine. Within the limitations of this procedure, we determined that this lower region (below $310 \mathrm{~m}$ ) is virtually uniform in its electromagnetic attenuation properties, with an attenuation rate of approximately $0.15 \mathrm{~Np} / \mathrm{m}$ at $21 \mathrm{MHz}$. This is an attenuation rate about one-third that from the data at $450 \mathrm{MHz}$ taken closer to the mine. A larger attenuation rate at higher frequencies is typically observed ${ }^{12-14}$ for in situ conditions. Thus, this observed variation of attenuation with frequency (i.e., dispersion) is consistent with expected behavior. Future site sfudies should assess the variation of attenuation with frequency for the same region. 


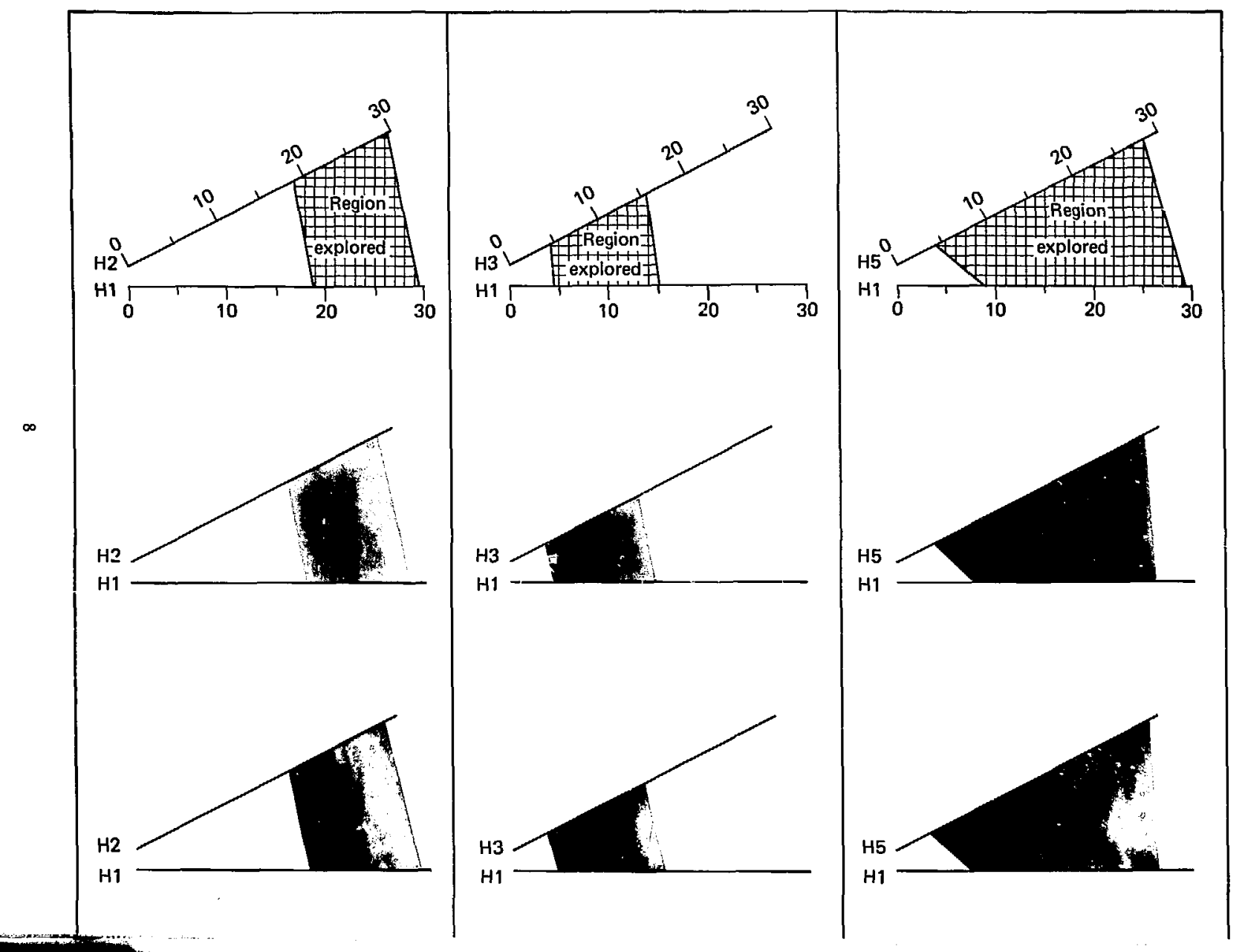



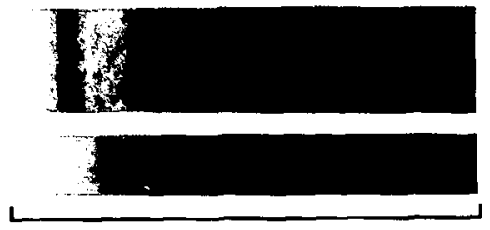

0

$\mathrm{Np} / \mathrm{m}$

1.0

F1G. 7. The planar sectors between boreholes that were sampled-H1-H2, H1H3, H1-H5, H2-H3, and H2-H5-and the pseudo-color and gray-level depictions of the geotomographs for the sampled sectors. (The planar secțors between boreholes $\mathrm{H3}-\mathrm{H} 4, \mathrm{H1}$ H4, and H4-H5 were nat sampled.) The numerical scales give slant-line distances from the mine face in meters. The pseudo-color and gray-level calibration representations are shown directly above this caption. Note that the electromagnetic attenuation rate is almost uniform in the H1-H2 and H2-H5 regions, which are more than five tunnel diameters Irom the mine face, while in the other three sections the electromugnetic attenuation rate is higher close to the mine izce. For those who prefer coordinate graphs of data to pictorial images, graphs of the interpreted attenuation rate along boreholes $\mathrm{H} 1$ and $\mathrm{H} 2$ are shown in Fig. 8.
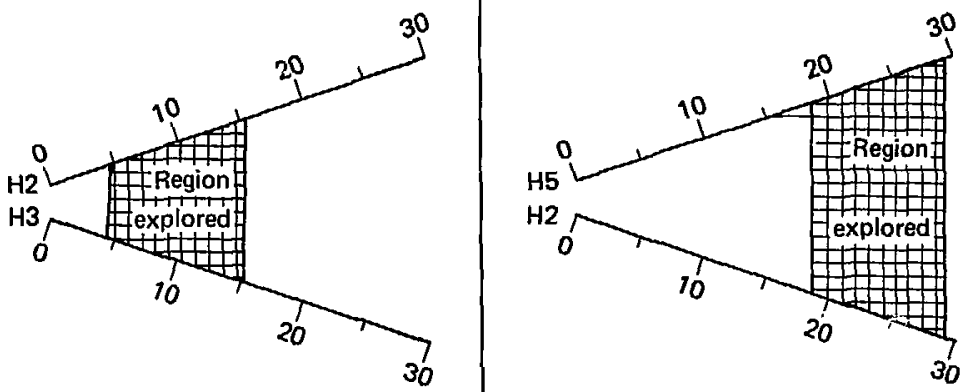

$\mathrm{H} 5$
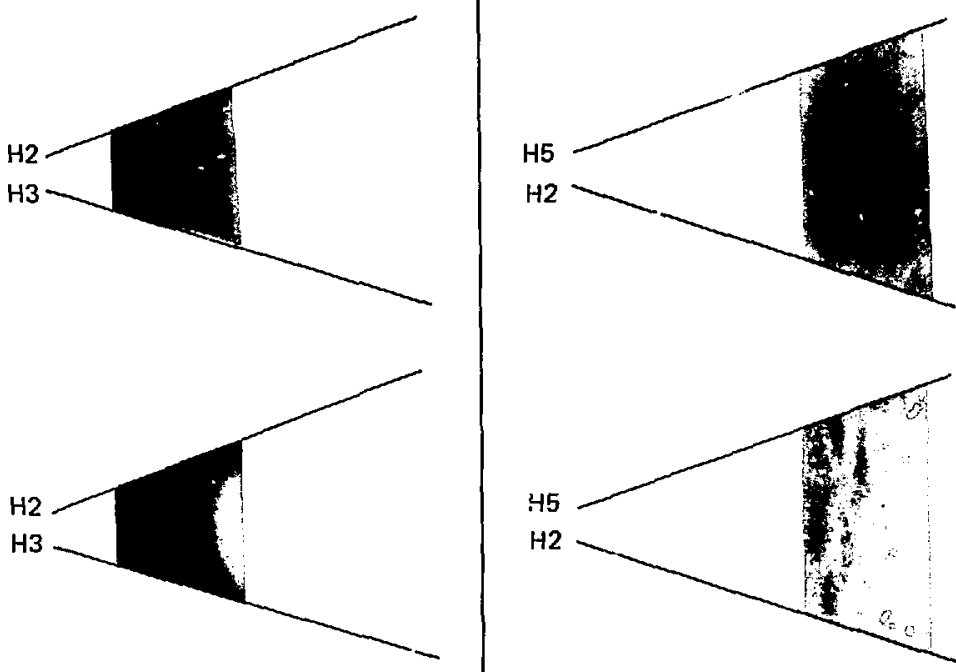

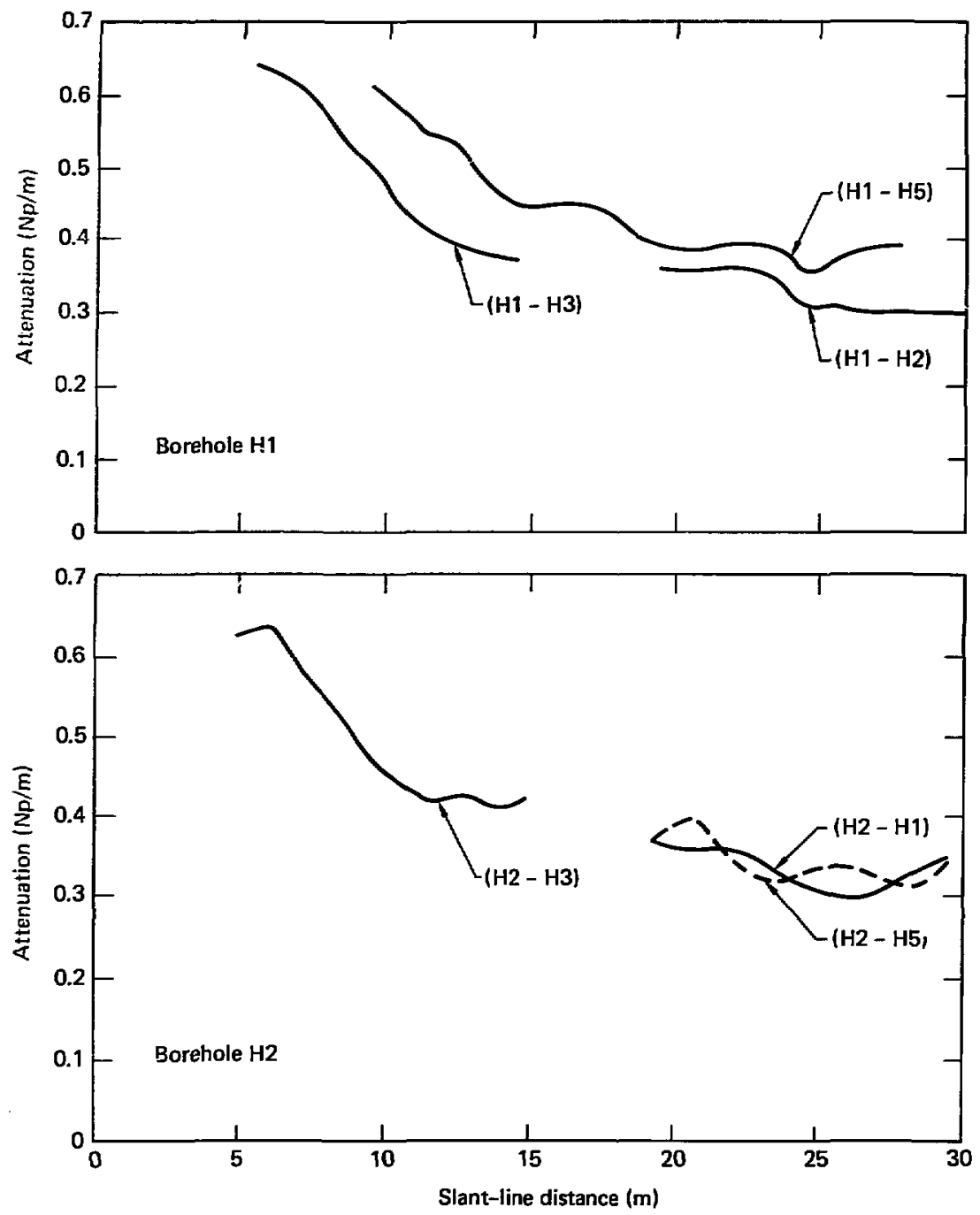

FIG. 8. The attemation mites stong borehole $\mathrm{HI}$ (top) and $\mathrm{H2}$ (bottom). The data sets are the same as shom alont these borcboles in Fig. 7. The inages in Fig. 7 are awother way of representing a large muber of sweh graphical presentations. 


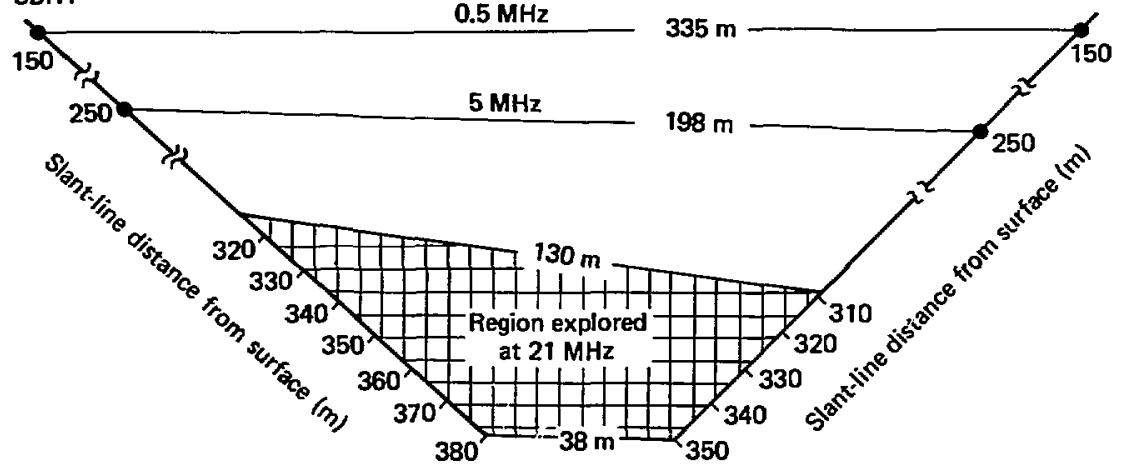

FIG. 9. Borcholes from the surface extended to the near vicinity of the mine. These boreholes are skewed relative to vertical and are not coplanor. The boretole designators are SBH1 and SBH2, standing for surface boreboles 1 and 2. Data were taken for frequencies of $0.5,5$, and $21 \mathrm{MHz}$.

\section{DATA INTERPRETATION}

The presence or absence of electromagnetically anomalous regions is visually evident in geotomographic images. If there is an anomalous region present in the image, all that is known from electromagnetic geotomography is that this local region has electromagnetic attenuation characteristies different from those in adjacent regions. A number of parameters can influence the electromagnetic attenuation. For example, possible inferences could be that the region may be a different geologic material from that of adjacent regions, ${ }^{15,16}$ the region may be of different fracture density, the region may be of different hydrologic character (c.g., different fluids, porosity, or permeability), the region may be at different $1 \mathrm{emperature,}{ }^{17,18}$ the region may be under different stress conditions, ${ }^{19}$ or the region may be ore-bearing or oil-bearing, 20 among other possibilities. Thus, if there are anomalies present in a geotomographic image, it is wise to use the geotomographic data together with all other available data to attempt to infer the reason for the anomalous region in the image.

Much like a detective attempting to solve a mystery or a physician attempting to diagnose a medical ailment, the interpreter of geotomographic data must use deductive reasoning to ascertain the degree of validity for each hypothesis about the site character. As in detective stories and in medical diagnoses, the hypothesis needs to be tested (sometimes at great risk and expense) to verify its validity.

The noted fictional detective Sherlock Holmes has been quoted as saying, when queried about a case, "I have devised seven separate explanations, each of which would cover the facts as far as we know them. But which of these is correct can only be determined by the fresh information which we shall no doubt find waiting for us." His method of solving the case is best presented by his statement-"Data, data, data! I can't make bricks without clay."21

Site characterization can be performed by using the same philosophical approach. By performing additional measurements (acquiring more data) complementary to the electromagnetic attenuation data, one can attempt to determine the correct site hypothesis. That is, one might hypothesize for a particular site that: the region imaged is gianite, with a fracture density described by an RQD of 25; the temperature is $10^{\circ} \mathrm{C}$ throughout the explored 
region: the conductivity of the fluid (water) in the rock (with $1 \%$ porosity) is $0.01 \mathrm{~S} / \mathrm{m}$; the mass density of the region is $2.50 \mathrm{~g} / \mathrm{cm}^{3}$ : and the major and minor principal stresses are respectively 18 and $12 \mathrm{MPa}$. The veracity of this particular hypothesis can be checked by comparing it with the known variation of all sitc data (including but not limited 10 geophysical, geochemical, rock mechanical, core sample. and geological data), available laboratory datil. theoretical studies, and experience. Alternat ive hypothests should also be formulated and an assessment performed of the veracity of each hyputhesis.

Within the available funds, we have altempted to apply such deduetive reasoning to the experimental datal obtained in Sweden. Hypotheses of phenomena which nay explain the electromagnetic attenuation data observed at the Stripa Site are: 1) fractures caused by blasting in creation of the mine; 2) hydrology perturbations ciused by creation of the mine; 3 ) stress relief caused by presence of the mine; 4) geological inhomogeneities; 5) some combination of the above: 6) some phenomenon not listed above. Using the limited additional data and funding available to us, we consider each of these hypoiheses below. Those having additional data and time to pursue this objective are encouraged to refine these hypotheses or suggest likely alternatives. We first consider the region near the mine, that is, the data collected by using boreholes H1, H2, H3, and $\mathrm{H} 5$.

Hypothesis 1 suggests that fractures caused by blasting in creation of the mine create the observed variation of electromagnetic attenuation with distance from the mine. Considering the care taken in blasting the rock within the mine drift where the measurements were taken, it is not expected that blasting created fractures extending to appreciable depths. Knowledgeable experts 22,23 estimate that blast-created fracture depths at Stripa do not extend berond one meter from the mine face. These experts qualify their best estimates by saying that there is a paucity of experimental data to confirm their estimate for the blasting technique used. Thus their estimates should be checked experimentally. For the present study, using the best information available at this time, hypothesis 1 has been rejected as the physical phenomenon causing the observed electricul losis gradient near the mine face.

Hypothesis 2 suggests that hydrological perturbations caused by creation of the mine create the observed variation of electromagnetic altenuation with distance from the mine. Considering the long time between creation of this mine drift and our experimental deployment, it is expected that there should (by hydrological conditions themselves) not nccessarily be more or less water relatively near the mine face than farther from the mine face. Hence, for the present study, on the basis of the best information available at this time, hypothesis 2 has been rejected as the physical phenomenon causing the observed behavior.

Hypothesis 3 suggests that stress relief caused by the presence of the mine creates the observed variation of electromagnetic attenuation with distance from the mine. This phenomenon has certain aspects that may be part of the observed behavior. Certain geologic materials have a known relationship between electrical conductivity and in simu stress. ${ }^{19}$

The variation of electrical conductivity with changing stress is strongest for the lower stress values. ${ }^{16}$ The known stress relief ${ }^{2+-26}$ near the mine would create lower stress values near the mine. Thus, this hypothesis may have merit. It is acknowledged that the observed variation of electrical conductivity with stress has been seen only if there is sufficient moisture in the pores of the rock. Thus, to this extent, if this phenomenon is indeed that which is seen, the explanation would include both the stress relief and hydrological factors.

Hypothesis 4 is that compositional differences create the observed variation of electromagnetic attenuation with distance from the mine. This particular mine site was chosen for its relatively uniform geological conditions. These uniform conditions were especially chosen to be appropriate for evaluating experimental and instrumentation procedures in the uniform environments which are characteristic of those to be used for nuclear waste repositories. Hence, the geological data available at this time cause us to reject hypothesis 4 as the physical phenomenon causing the observed behavior.

Hypothesis 5 suggests that a combination of the above hypotheses creates the observed variation of electromagnetic attenuation with distance from the mine. As was noted in the discussion of hypothesis 3 . the observed phenomenon may be due to a combination of stress relief and hydrology near the drift; the stress relief eaused by the presence of the drift results in the opening of microcracks, and 
these are filled with water. To check this hypothesis, measurements should be made on cores from this arca of the Stripa mine on the variation of electrical conductivity with stress.

Hypothesis 6 suggests that some phenonenon not considered in hypotheses I through 5 creates the observed variation of electromagnetic altenuation with distance from the mine. This is a particularly difficult hypothesis to refute a priori. Unless definitive measurements validate one of the prior hypotheses, this hypothesis is still a likely cause. Hence, further study is required to substantiate which hypothesis actually accounts for the observed variation of electromagnetic attenuation with dislance from the mine.

Regarding the region explored between surface horeholes 1 and 2, similar hypotheses need to be considered. In view of the large distance of this explored region from the mine, hypotheses 1-3 are not likely to be the governing physical phenomena. Hypotheses 4-6 may be correct. Additional datil are needed to decide the governing physical phenomena.

\section{MODEL CALCULATION AND DISCUSSION OF RESULTS}

To confirm that microcracks filled with groundwater are responsible for the observed attenuation near the mine drift requires both experimental and theoretical investigations. An experimental investigation might involve measurements of the variation of electrical conductivity with stress for cores from this area of the Stripa mine. This is left for future studies. The electrical properties of the rock should be modeled, and its calculated attenuation characteristics compared with those measured. A theoretical investigation might involve modeling the effect of microcracks filled with groundwater on the electrical properties of the rock mass. Such modeling can yield information on the validity of this hypothesis and possibly a quantitative description of the microfractures near the drift. In this section, we present such a model and discuss its implications for the interpretation of the geolomographs.

Both modeling calculations and in situ measurements indicate that the subsurface stress field is perturbed by the presence of a tunnel. The stress can be changed by a factor of 3 within a few tunnel diameters. ${ }^{26}$ At $330 \mathrm{~m}$ depth, the approximate depth of our measurements, the meisured vertical stress is $9.8 \mathrm{MPa} .{ }^{27}$ Laboratory data indicate that porosity of Westerly granite is not significantly altered-i.e., new cracks created-by uniaxial stress below about $60 \mathrm{MPa}{ }^{28}$ Therefore, it is unlikely that stress relief near the drift creates new microfractures in the rock. It is possible, however, that microcracks already present, normally closed by the hydrostatic stress far from the tunnel, are opened by the stress field changes near the tunnel.
The porosity of a Stripa granite core measured in the laboratory would be indicative of the maximum porosity change due to stress relief. Measured porosities of cores from the mine range up to $1.32 \% .{ }^{29}$ To summarize, we consider the hypothesis that the normal stress field in the Stripa granite is altered by a few megapascals due to the presence of the drift. This stress change opens microcracks, changing the rock porosity by $1 \%$ to $2 \%$, and these cracks are groundwater saturated. This additional water may account for the increased electromagnetic attenuation near the drift. To assess this hypothesis, consider the following.

We model the rock near the drift using a theory for the dielectric properties of mixtures. ${ }^{30}$ In this model the rock is the host medium, with intrinsic real dielectric permittivity $\boldsymbol{C}_{H}$ and electrical conductivity $\sigma_{H}$ such that the total complex permittivity of the host medium is

$$
\epsilon_{H} \equiv \epsilon_{H}^{\prime}-j \frac{o_{H}}{\omega},
$$

where $\omega$ is the angular frequency. The water-filled microcracks are modeled as inclusions with intrinsic complex permittivity

$$
\epsilon_{I} \equiv \epsilon_{I}^{\prime}-j \epsilon_{I}^{\prime \prime}-i \frac{\sigma_{I}}{\omega} .
$$

In this relation the real and imaginary dielectric constants of the inclusion are $\epsilon_{j}^{\prime}$ and $\epsilon_{j}$, respectively, and the inclusion conductivity is $\sigma_{l}$. The inclusions are assumed to be identical, spheroidal in shape, and either aligned or randomly oriented. The bulk 
effective electrical properties of this mixture are required. Although no exact solution to this problem has been published, various approximate solutions are possible. These solutions are based on estimates of the average electric field within the inclusions. For low-volume concentrations of inclusions, and when the characteristic dimension of the inclusion is small compared with the wavelength in the medium, the bulk permittivity of the mixture, $\epsilon$, is

$$
\epsilon / \epsilon_{H}=1-35 \epsilon / \epsilon_{H} \frac{1-\epsilon_{I} / \epsilon_{H}}{2 \epsilon / \epsilon_{H}+\epsilon_{I} / \epsilon_{H}}
$$

when the inclusions are spherical. The inclusion volume fraction is 5 . When the inclusions are prolate ellipsoids of eccentricity $I$ (needles) with random orientation.

$$
\epsilon / \epsilon_{H}=1-\frac{1}{3} \zeta\left(1-\epsilon_{I} / \epsilon_{H}\right)\left(1+\frac{4 \epsilon / \epsilon_{H}}{\epsilon / \epsilon_{H}+\epsilon_{I} / \epsilon_{H}}\right),
$$

and when inclusions are oblate spheroids of eccentricity I (disks) with random orientation,

$$
\xi / \epsilon_{H}=1-\frac{1}{3} \zeta\left(1-\epsilon_{I} / \epsilon_{H}\right)\left(2+\epsilon / \epsilon_{I}\right) .
$$

All measurements using the boreholes from the mine were made at $450 \mathrm{MHz}$. The propagation factor a (reciprocal of the distance for which the field strength decays $101 / e=0.368$ its original value) is

$$
a=\frac{2 \pi}{\lambda_{0}}\left[\frac{1}{2} \frac{\epsilon^{\prime}}{\epsilon_{0}}\left(\sqrt{1+\tan ^{2} \delta}-1\right)\right]^{1 / 2},
$$

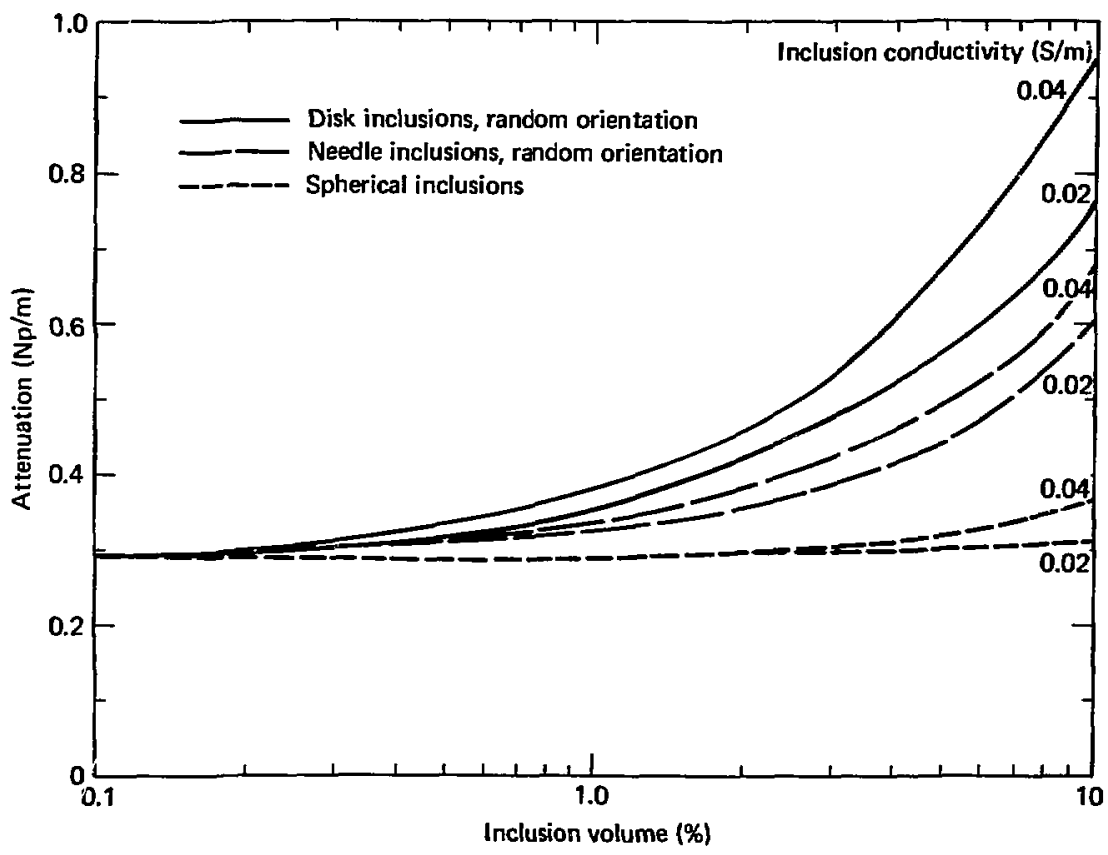

FIG. 10. Electromagaetic attenuation of the signal in a mixture, plotted as a function of the inclusion volume for varions types of indosions and inclusion combetivities of 0.2 and $0.4 \mathrm{~S} / \mathrm{m}$. The host conductivity is $0.0015 \mathrm{~S} / \mathrm{m}$. The experimentally determined attemation ranges from 0.3 to $0.7 \mathrm{~Np} / \mathrm{m}$, bet the expected porosity of the Stripa gramite is less than 2\%. Therefore, these models do not alequately explain the geotomographic data. 
where

$\lambda_{0}$ is the wavelength in free space. $\epsilon_{0}$ is the permittivity of free space, $\tan \delta=\left(\omega t^{\prime \prime}+\sigma\right) / \omega t^{*}$.

The transmitted power decays with a characteristic inverse length $2 \%$. The in situ measured dielectric constant is about 4 , and far from the tunnel $2 \alpha$ is 0.2 to $0.3 \mathrm{~Np} / \mathrm{m}$ (see Figs. 7 and 8). The measured groundwater electrical conductivity (direct current) varies, $\sigma_{I}=2-4 \times: 9^{-2} \mathrm{~S} / \mathrm{m}_{1}{ }^{31}$ and we use ${ }^{32,33}$

$$
\epsilon_{g}=80 \epsilon_{0}-j\left(0.45 \epsilon_{0}\right)-j \sigma_{I} / \omega .
$$

For these parameters, the calculated bulk attenuation for the Stripa granite is shown in Fig. 10. As expected, highly conductive water increases the effective rock conductivity and thus its attenuation, especially for porosities greater than a few tenths of a percent. However, these models do not explain attenuation ranging from $0.7 \mathrm{~Np} / \mathrm{m}$ near the drift to $0.3 \mathrm{~Np} / \mathrm{m}$ far from the drift (see Figs. 7 and 8) unless the porosity is $4-5 \%$ or the water conductivity is approxinately $10^{-1} \mathrm{~S} / \mathrm{m}$. Groundwater conductivity this high is not expected, since direct current measurements on water from the mine were not higher than $4 \times 10^{-2} \mathrm{~S} / \mathrm{m}^{31}$ It is also doubtful that the rock porosity is as high as $4-5 \%$, since core samples had porosities less than $1.3 \%$ and stresses at $330 \mathrm{~m}$ depth should not create new microfractures. 28,29

One possibility is that the stress field results in microcracks with a prcferred oricntation. If diskshaped cracks are oriented with their major axes perpendicular to the electric vector, the effective permittivity $\epsilon$ is

$$
\epsilon / \epsilon_{H}=I-\zeta\left(I-\epsilon_{I} / \epsilon_{H}\right)
$$

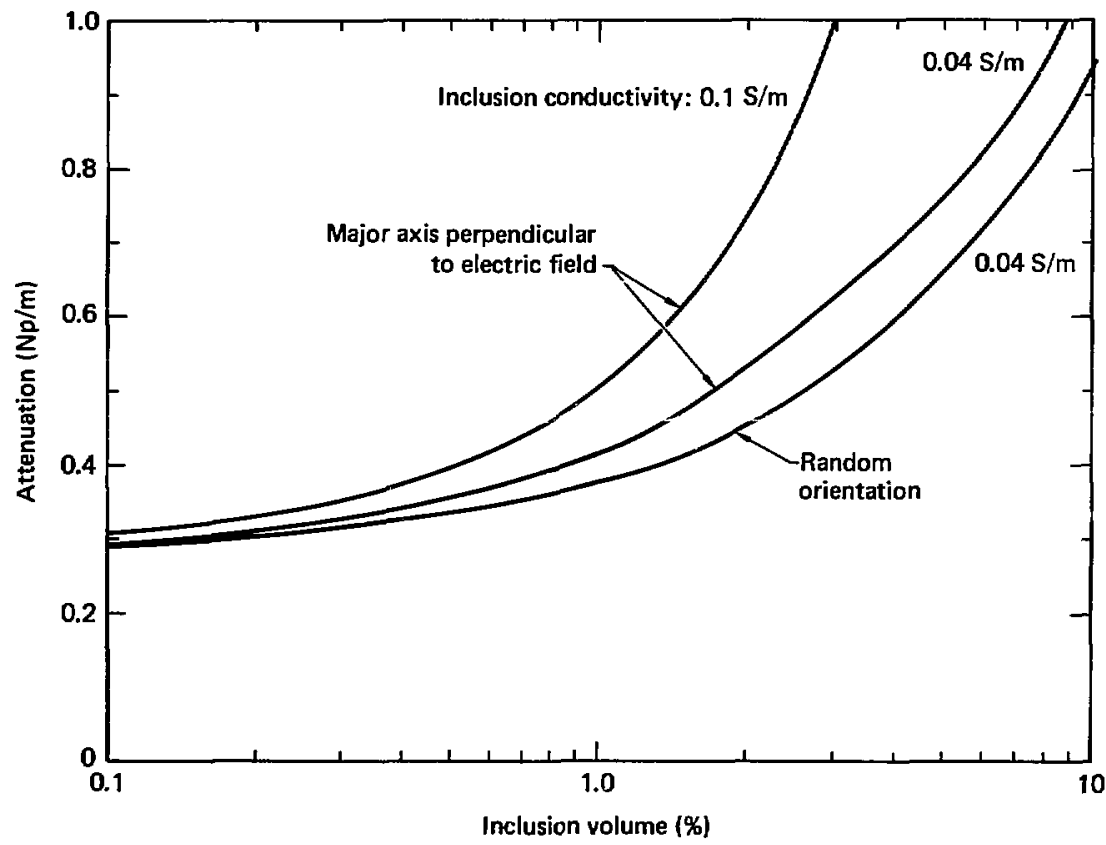

FIG. 11. Electromagnetic attemation of a signal in a mixture, plotted as a function of the inclusion volume for Alsk inclusions with random end aligned oricutation relative to the electric vector of the signal. The host conductivity is $0.0015 \mathrm{~S} / \mathrm{m}$. Omly when the inclusions are aligned perpendicular to the field and have a conductivity of 0.1 $S / m$ does the model accomet for the measured attencition rates of $0.7 \mathrm{~Np} / \mathrm{m}$ for porosities of approximately $2 \%$. 
which results in a larger attenuation for the mixture than for random orientation (see Fig. 11). Even this case, however, does not explain the larger attenuation rates measured near the drift. Other aljgnments of disk- and needle-shaped cracks result in smaller attenuation rates.

In summary, while these model calculations agree qualitatively with clectromagnetic properties near the drift, quantitative agrcement is not satisfactory. An enhunced porosity of $3-4 \%$ near the tunnel, due to stress relief of fracturing over several years or due to mining operations, would explain the phenomenon. Of course, a combination of two or more ejiects, or a mechanisin we have not considered, may be responsible for the variation of electromagnetic attenuation near the drift. Further studies, including modeling and laboratory measurements, are required to understand the phenomenon.

\section{CONCLUSIONS AND RECOMMENDATIONS}

In the course of this work, it was evident that the technology of geophysical tumography (geotomography) has significant merit for use in the problem of assessing a prospective underground nuclear waste repository. It also became evident that this technology would likely be appropriate for remote long-term monitoring of the repository site. Consequently, we strongly recommend the advancement of the state of the art of this technology, which is now in its infancy, for its needed application to both prospective site assessment and long-term remote monitoring.

In addition, we foresee the wisdom of obtaining seismic probing data complementary to the electromagnetic atienuation data. It is well known that an anomalous region can be revealed as a distinct geophysical contrast by seismic or electromagnetic waves. However, one cannot predict a priori which technique will detect the anomalous region. To ensure that geophysical techniques are effectively utilized for assessing whether a site has anomalous regions, both electromagnetic and seismic probing should be performed using the geotomographic approach.

This report has shown that the propagation properties of electromagnetic waves may be indicative of material properties that might not necessarily be considered electromagnetic in origin. That is, the relationship between in situ stress and electromagnetic attenuation is not especially well known nor has it been extensively used to interpret field measurements. Also not especially well known is the relationship between acoustic velocity and in situ stress. Nevertheless, the material properties in situ electromagnetic attenuation ${ }^{34,35}$ and acoustic velocity ${ }^{36,37}$ have been related to the in siru stress, which is a mechanical property, not an acoustic or clectromagnetic property.

The interrelationship of various material properties with remote probing observables is well known and much exploited in the realm of nondestructive evaluation of structural materials. To some extent, the interrelationship is also known, but not greatly exploited, in the evaluation of geological material. ${ }^{38}$ The range of interrelationships should be studied. For example, refined experimental measurements in the laboratory relating the attenuation of electromagnetic waves to stress, fluid conductivity, and geologic character are possible and desirable. Such empirical data would enable one to establish whether the phenomena observed in the vicinity of boreholes $\mathrm{HI}$ and $\mathrm{H} 5$ is likely due to the stress relief caused by the tunnel. The authors strongly recommend that geotomography be used in as wide a range of applications as possible. It appears that instrumentation systems can be built for sensing, among possible other phenomena of interest, in situ stress and fluid fow ${ }^{39}$ to a degree that is not presently done. These new systems should be built, tested, and routinely used if shown to be applicable. 


\section{ACKNOWLEDGMENTS}

The authors have benefited from informative discussions with personnel of Lawrence Berkeley Laboratory. In particular, Phil Nelson, LBL geophysicist, ably coordinated our activities with a number of other researchers and made a number of helpful observations regarding our experimental procedure. We wish to thank Hans Carlsson, site manager at Stripa, for his help and cooperation. The staff of Stallbergsbolagen made the stay in Sweden enjoyable and fruitful. In particular, Per Axel Halen, the mine supervisor, competently interacted with us. With the aid of all these people, we were able to complete all of our experimental objectives.

Colleagues of ours at LLNL provided helpful suggestions and fucilitated our experimental and interpretive efforts. These colleagues included; L. L. Cleland, K. A. Dines, R. W. Egbert, E. H. Fleming, E. A. Lafranchi, D. L. Lager, and E. K. Miller. The assistance of the Office of Nuclear Waste Isolation, Battelle Memorial Institute, working for the U.S. Department of Energy, made this work possible. 


\section{REFERENCES}

1. Lawrence Berkeley Laboratory information brochure on the cooperative effort.

2. P.H. Nelson, "Geophysical Program at Experimental Waste Storage Site at Stripa, Sweden," paper presented at the European Association of Exploration Geophysicists, Dublin, Ireland, June 1978.

3. D.L. Lager and R.J. Lytle, "Determining a Subsurface Electromagnetic Profile from Hligh-Frequency Measurements by Applying Reconstruction-Technique Algorithms," Radio Science 12(2), 249-260 (March-April 1977).

. 4. K.A. Dines and R.J. Lytle, "Computerized Geophysical Tomography," Proc. IEEE 67(7), 1065-1073 (July 1979 special issue on Exploration Geophysics).

5. R.J. Lytle. "Geophysical Characterization Using Advanced Data Processing," invited paper published in Proc. NSF Site Characterization Workshop, Evanston, Illinois, June 12-14, 1978 (available from the American Society of Civil Engineers, 345 East 47th Street, New York, NY 10017).

6. R.J. Lytle, "Cross-Borehole Geophysical Probing for Site Characterization," Proc. 1979 Rapid Excavation and Tumneling Conference of the Society of Mining Engineers of the AIME, Atlanta, Georgia, June 1979, vol. 1, pp. 87-96. Also, Lawrence Livermore National Laboratory report UCRL-81901 Preprint (1979).

7. R.J. Lytle, K.A. Dines, E.F. Laine, and D.L. Lager, Electromagnetic Cross-Borehole Survey of a Site Proposed for an Urban Transit Station, Lawrence Livermore National Laboratory report UCRL-52484 (1978).

8. E.F. Laine, K.A. Dines, J.T. Okada, and R.J. Lytle, Probing Concrete with Radio Waves, Lawrence Livermore National Laboratory report UCRL-82042 Preprint (1979) (to be published in $A S C E J$. Geotech. Eng. Div.).

9. E.F. Laine, R.J. Lytle, and J.T. Okada, Cross-Borehole Obserwation of Soil Grouting, Lawrence Livermore National Laboratory report UCRL-82543 Preprint (1979) (to be published in ASCE J. Geotech. Eng. Dis.).

10. R.N. Grubb and J.R. Wait, "In-Situ Measurements of the Complex Propagation Constant in Rocks for Frequencies from $1 \mathrm{MHz}$ to $10 \mathrm{MHz}$," Electron. Lett. 7(17), 506-507 (1971).

11. J.R. Wait and J.A. Fuller, "On Radio Propagation Through Earth," IEEE Trans. Antennas Propag. AP19, 796-798 (1971).

12. J.C. Cook, "Radar Transparencies of Mine and Tunnel Rocks," Geophysics 40(5), 865-885 (October 1975).

13. J.A. Fuller and J.R. Wait, "A Pulsed Dipole in the Earth," ch. 5 in Transient Electromagnetic Waves, L.B. Felsen, ed. (Springer-Verlag, Berlin, 1976).

14. R.T. Shuey and M. Johnson, "On the Phenomenology of Electrical Relaxation in Rocks," Geophysics 38(I), 37-48 (1973).

15. P. Hoekstra and A. Delaney, "Dielectric Properties of Soils at UHF and Microwave Frequencies," $J$. Geophy's. Res. 79(11), 1699-1708 (April 1974).

16. G.R. Olhoeft, "Electrical Properties of Rocks," in The Physics and Chemistry of Minerals and Rocks, R.G.J. Strens, ed. (John Wiley, New York, 1976), pp. 261-278.

17. A. Duba and A.E. Ringwood, "Electrical Conductivity, Internal Temperatures and Thermal Evolution of the Moon," The Moon 7 (D. Reidel Publishing Company, Dordrecht, 1973), pp. 356-376.

18. A.G. Duba, "Electrical Conductivity of Coal and Coal Char," Fuel 56, 44I-443 (1977).

19. E.I. Parkhomenko, Electrical Properties of Rocks, translated from the Russian by G. V. Keller (Plenum Press, New York, 1967).

20. A.J. Piwinskii and A. Duba, "The Permittivity and Electrical Conductivity of Oil Shale," Int. J. Rock Mech. Min. Sci. Geomech. Abstr. 13, 165-166 (1976).

21. Selected quotations from A. Conan Doyle, "The Adventure of the Copper Beeches," in The Complete Sherlock Holmes (Doubleday, New York, 1930).

22. Prof. N.G.W. Cook, University of California, Berkeley, Department of Materials Science and Mineral Engineering, private communication. 
23. R. Langland, Lawrence Livermore National Laboratory, private communication.

24. J. C. Jaeger, Rock Mechanics and Engineering (Cambridge University Press, 1972).

25. C. Jaeger and N.G.W. Cook, Fundamentals of Rock Mechanics (Methuen \& Co. Ltd., London, 1969).

26. R.E. Goodman, "On the Distribution of Stresses around Tunnels in Nonhomogencous Rocks," Proc. First Congress International Society of Rock Mechanics, Lisbon, vol. 2, 249-255 (1966).

27. H. Carlson, Stress Measurements in the Stripa Granite, Lawrence Berkeley Laboratory, Berkcley, California, report LBL-7078, SAC-04 (1978).

28. H.C. Heard, A.E. Abey, B.P. Bonner, and R.N. Schock, Mechanical Behavior of Dry Westerly Granite at High Pressure, Lawrence Livermore National Laboratory report UCRL.5I642 (1974).

29. P. Neison, B. Paulson, R. Rachiele, L. Anderson, T. Schraul, W. Hustruliel, O. Durun, and K.A. Magnusson, Preliminary Report on Geophysical and Mechanical Borehole Measurements at Stripa, Lawrence Berkeley Laboratory, Berkeley, California, report LBL-8280, SAC-16 (1979).

30. L.S. Taylor, "Dielectric Properties of Mixtures," IEEE Trans. Antermas Propag. AP-13(6), 943-947 (1965).

31. P. Fritz, J.F. Barker, and J.E. Gale, Geochemistry and lsotope Hydrology of Groundwaters in the Stripa Granite-Results and Preliminary Interpretation, Lawrence Berkeley Laboratory, Berkeicy, California, report LBL-8285, SAC-12 (1979).

32. D. Eisenberg and W. Kauzman, The Structure and Properties of Water (Oxford University Press, New York, 1969).

33. N. E. Hill, "Theoretical Treatment of Permittivity and Loss," in Dielectric Properties and Molecular Behavior, T. M. Sugden, ed. (Van Nostrand, Lundon, 1969).

34. A. Mazzella and H.F. Morrison, "Electrical Resistivity Variations Associated with Earthquakes on the San Andreas Fault," Science 185, 855-857 (September 6, 1974).

35. Y. Yamazaki, "Electrical Conductivity of Strained Rocks, the First Paper: Laboratory Experiments on Sedimentary Rocks," Bull. Earthquake Res. Inst. 43, 783-802 (1965); "Electrical Conductivity of Strained Rocks, the Second Paper: Further Experiments on Sedimentary Rocks," Bull. Earthquake Res. Inst. 44, 1553-1570 (1966); "Electrical Conductivity of Strained Rocks, the Third Paper: A Resistivity Variometer," Bull. Earthquake Res. Inst. 45, 849-860 (1967).

36. G.S. Kino, J.B. Hunter, G.C. Johnson, A.R. Selfrilge, D.M. Barnet, G. Hermann, and C.R. Steele, “Acoustoelastic Imaging of Stress Fieids," J. Appl. Phys. 40(4), 2607-2613 (April 1979).

37. R.N. Schock, B.P. Bonner, and H. Louis, Collection of Ultrasonic Velocity Data as a Function of Pressure for Polycrystalline Solids, Lawrence Livermore National Laboratory report UCRL-51508 (1974).

38. R.E. Goodman, Methods of Geological Engineering in Discontinuous Rocks (West Publishing Co., New York, 1976), p. 32.

39. R.J. Lytle, D.L. Lager, E.F. Laine, J.D. Salisbury, and J.T. Okada, Fluid Flow Monitoring Using Electromagnetic Probing, Lawrence Livermore National Laboratory report UCRL-83444 Preprint (1979). 


\section{APPENDIX :}

\section{PHOTO ALBUM}

For thase who have never had the pleasure of conducting a geophysical diagnostics experiment, we bave included a few photos to show the working environment in Sweden. The Stripa Mine once produced iron orc. It is now heing used as a test site for determining information related to the underground storage of nuclear wastes. While in Sweden, the researchers stayed at the scenie "Stripa Hilton," a harracks within walking distance of the mine. The good company, resident technical expertise, and pleasant surroundings were enjoyed by the experimenters during their live-wesk sojourn.

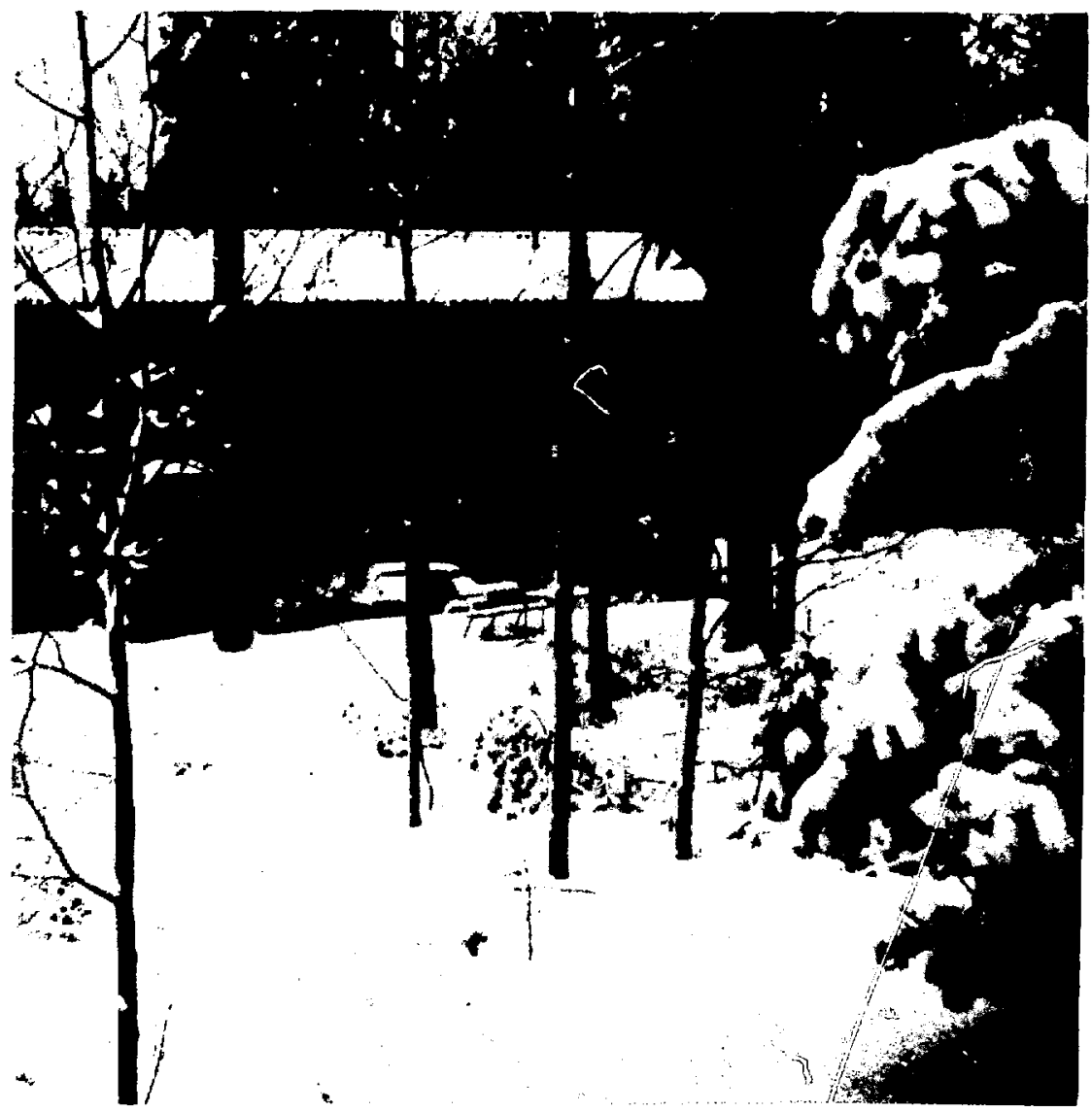


Existing above-surface mine buildings are shown here in this photo of LLNL workers walking to work early in the morning.
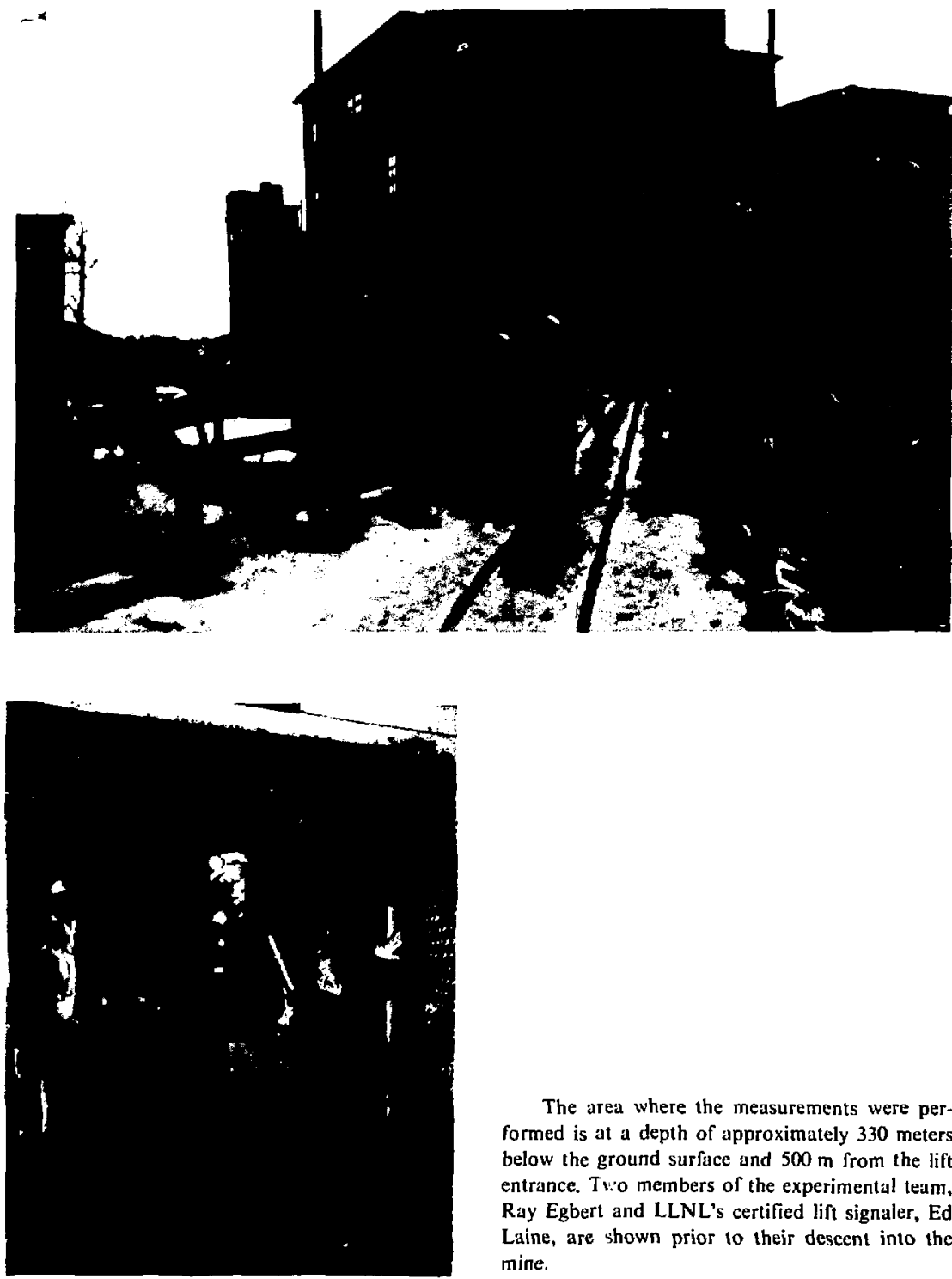

The area where the measurements were performed is at a depth of approximately 330 meters below the ground surface and $500 \mathrm{~m}$ from the lift entrunce. Tr:o members of the experimental team, Ray Egbert and LLNL's certified lift signaler, Ed Laine, are shown prior to their descent into the mine. 
The working environment in the mine was cool (approximately $12^{\circ} \mathrm{C}$ ) with adequate ventilation. The physical situation at the cnd of the drift where the extensive cross-borehole tests were performed is shown here. This is the end of the drift in the upper right-hand corner of Fig. 5 in the body of the report.

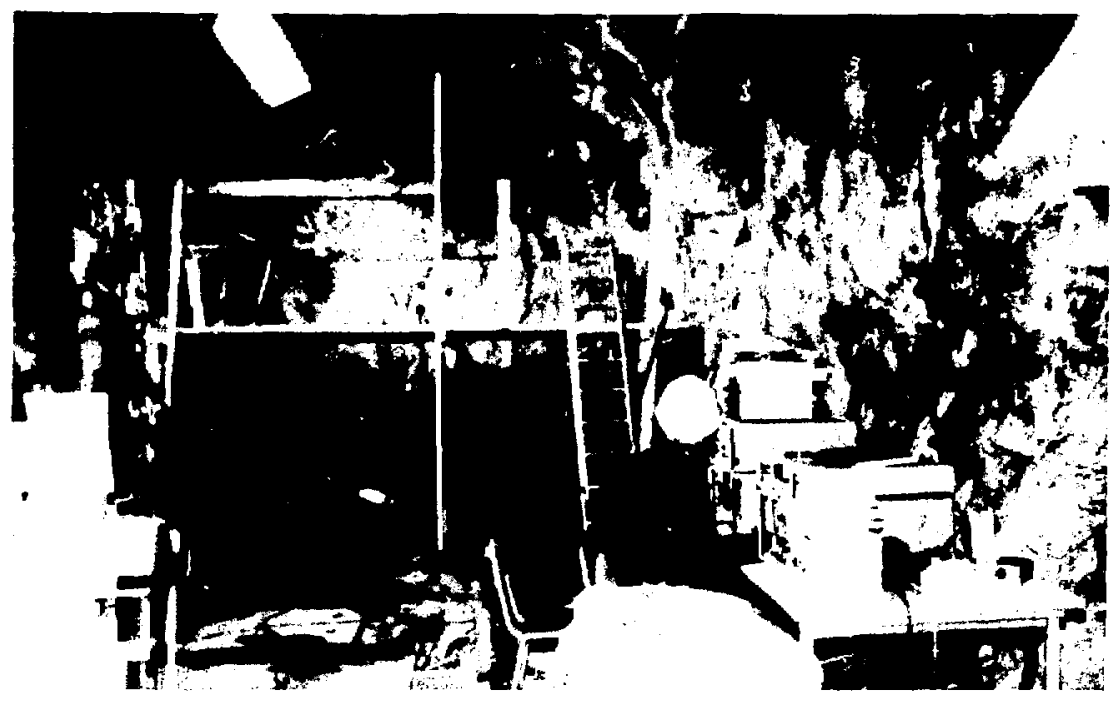

Electromagnetic sensors and sources were manually positioned at over 25,000 distinct locations using the technique illusirated by Ray Egbert.

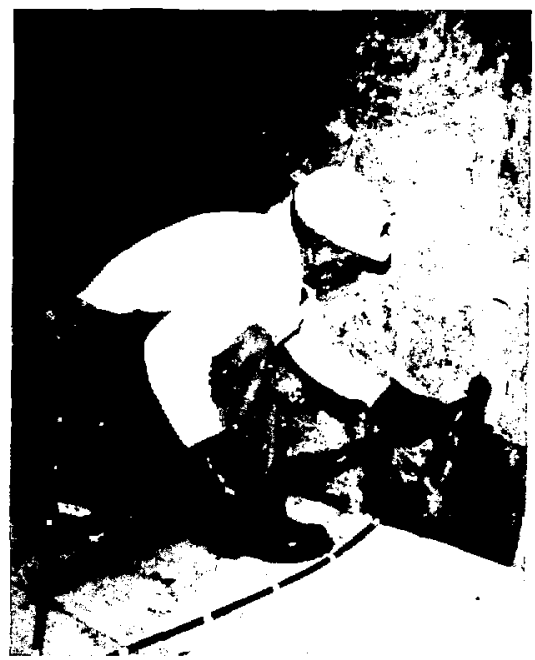


By using electronic instrumentation, each of the over 25,000 data points was visually ascertained and manually recorded in a $\log$ book. Justin Okada is shown performing this operation.

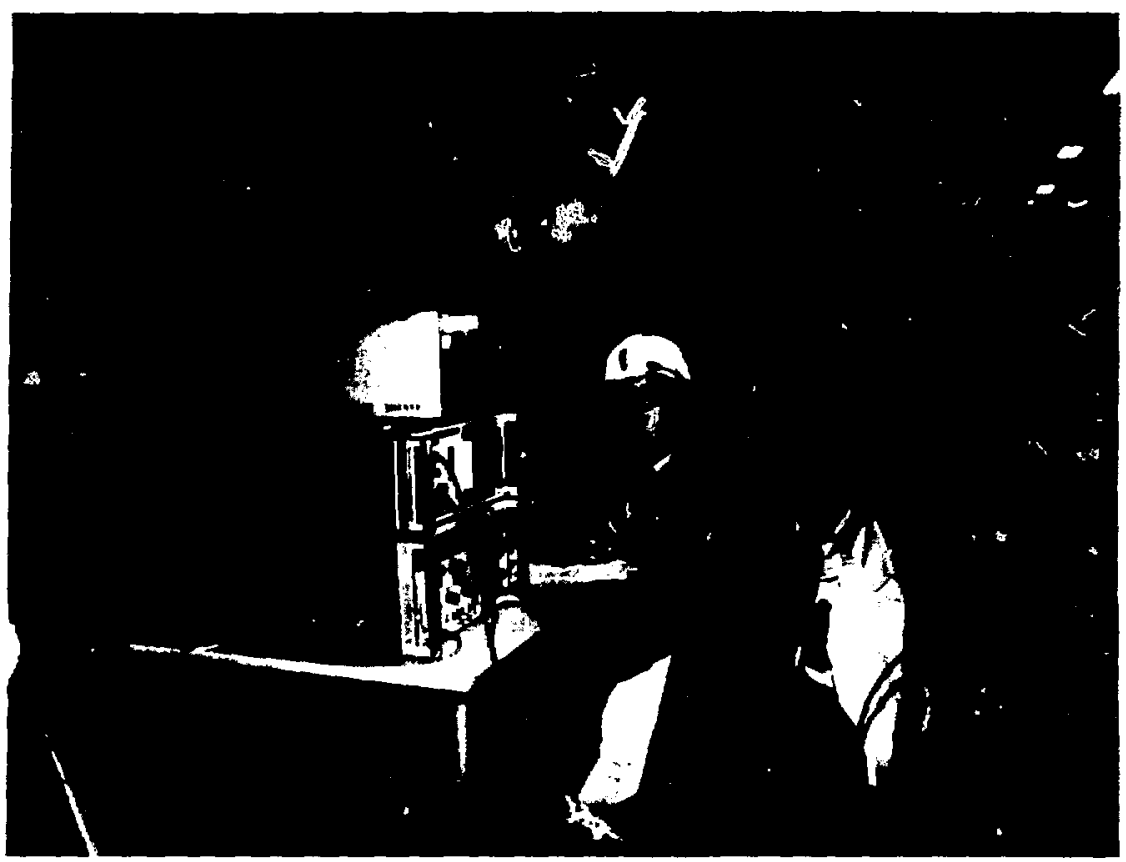

As usual, those who became intimately familiar with this data collection/recording scenario proposed ways the system could be significantly improved. Upon our return to LLNL, the data were entered into the CDC-7600 computer memory and processed to provide the results discussed in this report. The use of such a large-scale computer system is, however, not required to perform the data processing. In fact. Justin Okada demonstrated that it was possible to suitubly process the data in the field. He did this for a planar cross-section between two boreholes by using the MODCOMP computer resident in the mine for recording and processing other instrumentation data. 